\title{
Saikosaponin-d inhibits proliferation of DU145 human prostate cancer cells by inducing apoptosis and arresting the cell cycle at G0/G1 phase
}

\author{
MIN YAO, JINGBO YANG, LANQING CAO, LIAN ZHANG, SHANSHAN QU and HONGWEN GAO \\ Department of Pathology, Jilin University Bethune Second Hospital, Changchun, Jilin 130041, P.R. China
}

Received August 27, 2013; Accepted March 12, 2014

DOI: $10.3892 / \mathrm{mmr} .2014 .2153$

\begin{abstract}
Saikosaponin-d (SSd), a triterpene saponin compound derived from Bupleurum radix, has been shown to have a cytotoxic effect on various cancer cell lines. However, its effect on prostate cancer cells has remained unexplored. The present study reports the apoptosis-inducing effect of SSd on the DU145 human prostate carcinoma cell line. Treatment with SSd inhibited DU145 cell proliferation in a concentration-dependent manner. Flow cytometric analysis showed that SSd inhibited the proliferation of DU145 cells by induction of apoptosis and cell cycle arrest at G0/G1 phase. Further mechanistic experiments demonstrated that SSd arrested the cell cycle at G0/G1 phase via upregulation of p53 and p21 and induced apoptosis by modulating B-cell lymphoma 2 family proteins, dissipation of the mitochondrial membrane potential, release of cytochrome $c$ into the cytosol and activation of caspase-3. In conclusion the present study indicated that $\mathrm{SSd}$ induced apoptosis in DU145 cells by the intrinsic apoptotic pathway. Therefore, SSd may become a leading candidate drug for the therapy of prostate carcinoma.
\end{abstract}

\section{Introduction}

Saikosaponins (SSs) are triterpene saponins isolated from the root of Bupleurum falcatum L. (Umbelliferae) (1). As all SSs have a common steroid-like structure, they were expected and also confirmed to exert a number of steroid-associated pharmacological activities $(2,3)$. SSs are also regarded as the major effective components of xiao-chai-hu-tang, one of the most popular Chinese medicinal formulae that has been widely used for its various pharmacological effects, including anti-inflammatory, antioxidant and antihepatic fibrosis proper-

Correspondence to: Professor Hongwen Gao, Department of Pathology, Jilin University Bethune Second Hospital, 218 Ziqiang Street, Changchun, Jilin 130041, P.R. China

E-mail: gaohongwen@jlu.edu.cn

Key words: saikosaponin-d, DU145 cells, anticancer, apoptosis, $\mathrm{G} 0 / \mathrm{G} 1$ phase arrest ties (4-6). Thus far, at least 10 types of SS have been identified, and among which saikosaponin-d (SSd) is considered to be the most active component (1,7). Studies have reported that SS may potently inhibit the proliferation of hepatocellular carcinoma (8), cervical cancer (9), lung adenocarcinoma (10), colon carcinoma (11), breast cancer (12) and melanoma (13) cells. However, the effect of SS on human prostate cancer cell lines remains to be elucidated.

Prostate cancer is one of the most commonly diagnosed cancers among males, and is the second most common cause of cancer mortalities in developed countries (14). With the developments in diagnosis and therapy, the mortality rate of prostate cancer has decreased significantly (15-18). Although prostate cancers are initially treatable, the androgen-insensitive or hormone-refractory recurrent cases of prostate cancer are not responsive to current therapies. Therefore, there is an urgent requirement for novel therapeutic agents.

In the present study, the anti-proliferative effects and associated mechanisms of SSd on the DU145 human prostate cancer cell line were investigated for the first time, to the best of our knowledge.

\section{Materials and methods}

Reagents. SSd was obtained from the National Institute for the Control of Pharmaceutical and Biological Products (Beijing, China). Dimethyl sulfoxide (DMSO) was purchased from Sangon Biotech (Shanghai) Co., Ltd. (Shanghai, China). Rabbit anti-human cleaved-caspase-3, Bcl-2-associated $\mathrm{X}$ protein (Bax), p21, p53, cytochrome-c, mouse anti-human B-cell lymphoma 2 (Bcl-2) and $\beta$-actin primary antibodies were purchased from Cell Signaling Technology, Inc. (Shanghai, China). Horseradish peroxidase-conjugated secondary antibodies (anti-mouse and anti-rabbit) were purchased from Santa Cruz Biotechnology, Inc. (Beijing, China). Trypsin, Hoechst 33258, Rhodamine 123 (Rho-123), penicillin and streptomycin were purchased from Sigma-Aldrich (Beijing, China).

Cell culture. The DU145 human prostate cancer cell line (ATCC HTB-81) was obtained from the American Type Culture Collection (Manassas, VA, USA), and cultured in Dulbecco's modified Eagle's medium (Sigma-Aldrich) supplemented with $10 \%$ fetal bovine serum (HyClone Laboratories, Inc., Logan, 
UT, USA), $100 \mathrm{U} / \mathrm{ml}$ penicillin and $100 \mu \mathrm{g} / \mathrm{ml}$ streptomycin in a $\mathrm{CO}_{2}$ incubator $\left(37^{\circ} \mathrm{C}, 5 \% \mathrm{CO}_{2}, 95 \%\right.$ humidity).

MTT assay. Cell viability was determined by an MTT assay as previously described (19). Briefly, $100 \mu 1$ cell suspension $\left(1 \times 10^{5}\right.$ cells) was seeded in $96-$ well plates and incubated for $12 \mathrm{~h}$, and then the cells were exposed to $\mathrm{SSd}(0,1,2.5,5$, 10,20 or $50 \mu \mathrm{M}$ ) for $24 \mathrm{~h}$. Following treatment, $20 \mu \mathrm{l}$ MTT $(5 \mathrm{mg} / \mathrm{ml})$ was added and the cells were incubated at $37^{\circ} \mathrm{C}$ for $4 \mathrm{~h}$. The culture medium was removed and $150 \mu \mathrm{l}$ DMSO was added to each well to dissolve the formazan crystals. The absorbance $(570 \mathrm{~nm})$ was measured using a microplate reader (Varioskan Flash; Thermo Fisher Scientific, Waltham, MA, USA). The percentage of viable cells was determined using the following formula: Cell viability $(\%)=(\mathrm{A} 570$ treated $/ \mathrm{A} 570$ control) x 100; and the $\mathrm{IC}_{50}$-values were calculated using GraphPad Prism, version 5 (GraphPad Software, Inc., La Jolla, CA, USA).

Cellular morphological changes. DU145 cells were incubated with different concentrations $(3,9$ or $15 \mu \mathrm{M})$ of SSd for $24 \mathrm{~h}$. Morphological changes of the cells were visualized under a phase contrast microscope (1x71; Olympus Corporation, Tokyo, Japan), recorded with a charge-coupled device (CCD) camera (DP72; Olympus Corporation) and analyzed using DP2-BSW software, version 2.2 (Olympus Corporation). For mouse splenocyte morphological study, the cells were analyzed by trypan blue $(0.4 \%)$ staining prior to the microscopic visualization.

Flow cytometric analysis of apoptosis. Cell apoptosis was detected by flow cytometry using an Annexin V-fluorescein isothiocyanate (FITC) Apoptosis Detection kit (Beyotime Institute of Biotechnology, Shanghai, China). Briefly, DU145 cells cultured in six-well tissue culture plates were treated with different concentrations of SSd for $12 \mathrm{~h}$. Following treatment, the cells were collected and washed twice with ice-chilled phosphate-buffered saline (PBS). The cell pellets were stained with Annexin V-FITC and propidium iodide (PI) according to the manufacturer's instructions. Late apoptosis was defined as Annexin V-positive/PI-positive and early apoptosis was defined as Annexin V-positive/PI-negative as determined by flow cytometry (Epics XL; Beckman Coulter, Miami, FL, USA).

Nuclear staining. Following exposure to the test compound for $12 \mathrm{~h}$, the DU145 cells were harvested and washed twice with PBS. The cells were then stained with Hoechst $33258(50 \mu \mathrm{g} / \mathrm{ml})$ at $37^{\circ} \mathrm{C}$ for $10 \mathrm{~min}$ in the dark. After the staining, the cells were washed twice with PBS and analyzed using a fluorescence microscope $(1 \times 71$; Olympus Corporation) installed with a CCD camera (DP72; Olympus Corporation) and analyzed using DP2-BSW software (Olympus Corporation). Apoptotic cells were defined as cells exhibiting nuclear shrinkage and chromatin condensation.

Flow cytometric analysis of the cell cycle. DU145 cells were treated with different concentrations $(3,9$ and $15 \mu \mathrm{M})$ of SSd for $12 \mathrm{~h}$, trypsinized, washed twice with PBS and fixed in $70 \%$ ice-cold ethanol overnight. The fixed cells were rinsed twice with PBS and then stained with $50 \mu \mathrm{g} / \mathrm{ml}$ PI (containing
$100 \mu \mathrm{g} / \mathrm{ml}$ RNase A) using a Cell Cycle and Apoptosis Analysis kit (Beyotime Institute of Biotechnology) according to the manufacturer's instructions. Cell cycle phase distributions of nuclear DNA were assayed using flow cytometry (Epics XL; Beckman Coulter) and CellQuest software (BD Biosciences, Franklin Lakes, NJ, USA).

Reactive oxygen species (ROS) generation detection. DU145 cells cultured in six-well tissue culture plates were treated with different concentrations of SSd. The cells were then stained with $10 \mu \mathrm{mol} / 12^{\prime}, 7^{\prime}$-dichlorofluorescein-diacetate using a ROS Assay kit (Beyotime Institute of Biotechnology) according to the manufacturer's instructions. The cells were then collected, washed three times with PBS and assayed using flow cytometry (Epics XL; Beckman Coulter) as described previously (20).

Mitochondrial membrane potential (MMP) determination. DU145 cells were treated with different concentrations of SSd for $12 \mathrm{~h}$. The cells were trypsinized, collected in a centrifuge tube, and then stained with Rho- $123(10 \mu \mathrm{g} / \mathrm{ml})$ at $37^{\circ} \mathrm{C}$ for $30 \mathrm{~min}$ in the dark. Following staining, the cells were washed three times with PBS and assayed using flow cytometry (Epics XL; Beckman Coulter).

Western blot analysis. DU145 cells were treated with different concentrations of SSd for $12 \mathrm{~h}$ and then cell extracts were prepared using a Bicinchoninic Acid Protein Assay kit (Beyotime Institute of Biotechnology). The cell lysates (containing $40 \mu \mathrm{g}$ protein) were subjected to SDS-PAGE and analyzed by western blotting using various antibodies according to standard protocols. The proteins were visualized using an Enhanced Chemiluminescence Plus kit (Millipore Corporation, Billerica, MA, USA).

Statistical analysis. All data are expressed as the mean \pm standard error of the mean from at least three independent experiments. Statistical analysis was performed using Student's t-test. $\mathrm{P}<0.05$ was considered to indicate a statistically significant difference.

\section{Results}

Antiproliferative effect of SSd on DU145 human prostate carcinoma cells. The inhibitory effects of SSd (chemical structure is shown in Fig. 1A) on the proliferation of DU145 human prostate carcinoma cells were determined using an MTT assay. As shown in Fig. 1B, SSd treatment induced concentration-dependent proliferation inhibition of the DU145 cells. At $24 \mathrm{~h}$, maximal inhibition was achieved with $50 \mu \mathrm{M}$ SSd, which inhibited $80 \%$ of the DU145 cells proliferation, and the $\mathrm{IC}_{50}$-value of the inhibition was $\sim 10 \mu \mathrm{M}$. Morphological changes of the DU145 cells treated with SSd were visualized under a phase contrast microscope, which revealed a reduced number of adherent cells accompanying an increased number of floating cells in the culture medium compared with those in the culture medium containing the untreated cells (Fig. 1C). Furthermore, the effect of SSd on primarily cultured mouse splenocytes was investigated and trypan blue analysis indicated that SSd had little toxicity on the cells compared with the control cells (Fig. 1D). 
A

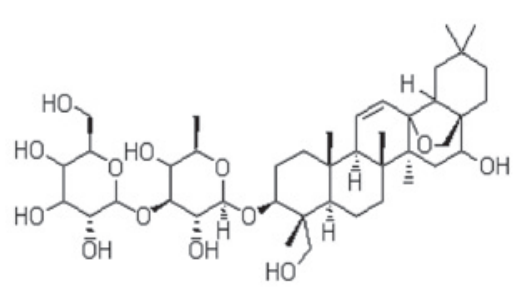

C
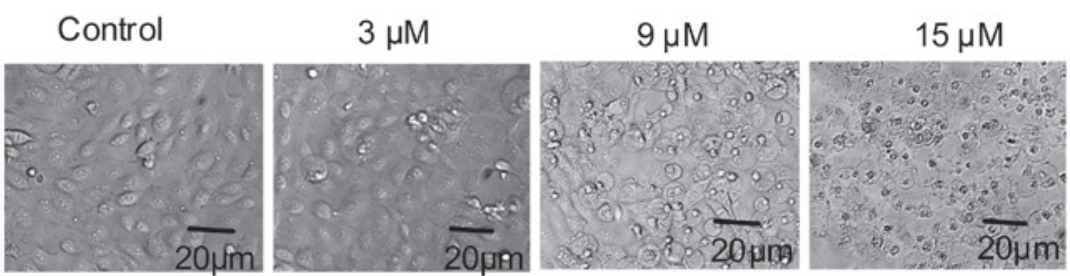

D
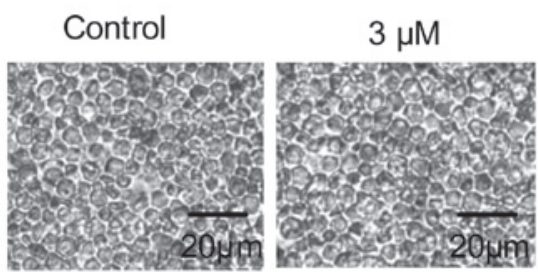

$9 \mu \mathrm{M}$

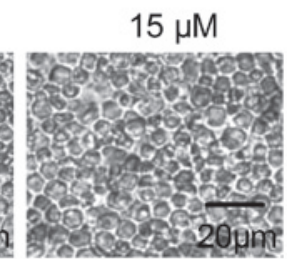

Figure 1. Effects of SSd on the viability and morphological characteristics of DU145 cells. (A) Chemical structure of SSd. (B) DU145 cells were treated with the indicated concentrations of SSd for $24 \mathrm{~h}$. The cell survival rate was measured using the MTT assay. Data are expressed as the mean \pm standard error of three independent experiments with similar results. Morphological changes of (C) DU145 cells and (D) mouse splenocytes observed under a phase contrast microscope after the cells were treated with dimethyl sulfoxide (negative control) or the indicated concentrations of $\mathrm{SSd}$. ${ }^{*} \mathrm{P}<0.05$; ${ }^{* *} \mathrm{P}<0.01$ compared with the control. Scale bar, $20 \mu \mathrm{m}$. Magnification, x200. SSd, saikosaponin-d.

Effects of SSd on cell cycle distribution. Cell cycle arrest and apoptosis are major causes of cell proliferation inhibition (20-22). To investigate the mechanisms responsible for SSd-induced inhibition of DU145 cell proliferation, the cell cycle distribution affected by SSd was measured. DU145 cells were exposed to different concentrations of SSd for $12 \mathrm{~h}$ and then the cell cycle distributions were determined using PI staining and flow cytometric analysis. The data indicated that SSd caused a significant accumulation of DU145 cells in G0/G1 phase compared with that of the control cells. Compared with that of the DMSO control, the proportions of cells in $\mathrm{G} 0 / \mathrm{G} 1$ phase were increased from 51.7 to 53.3, 56.8 and $60.3 \%$ following treatment with 3,9 and $15 \mu \mathrm{M} \mathrm{SSd}$, respectively (Fig. 2A). To elucidate the molecular mechanism underlying the G0/G1 phase arrest induced by SSd, several key proteins involved in the G1 phase transition were investigated in DU145 cells. The cells were treated with 3,9 and $15 \mu \mathrm{M}$ SSd for $12 \mathrm{~h}$ and the expression levels of $\mathrm{p} 53$ and p21 proteins were analyzed by western blotting. The data showed that SSd treatment significantly increased the expression levels of p53 and p21 compared with those of the control cells (Fig. 2B).

Effects of SSd on apoptosis induction. Subsequently, the effect of SSd on the induction of apoptosis of DU145 cells was investigated. Cell apoptosis, a type of programmed cell death, is characterized by nuclear condensation, cell shrinkage, membrane blebbing and DNA fragmentation (23), with nuclear condensation being a key characteristic (24). Morphological changes of the cell nuclei were observed using Hoechst 33258 staining. The results revealed that treatment of DU145 cells with SSd resulted in significant levels of nuclear condensation: 3,9 and $15 \mu \mathrm{M}$ SSd treatment increased the percentage of cleaved nuclei from $5.13 \pm 0.84$ (in the DMSO control group) to $10.01 \pm 1.71,24.50 \pm 1.82$ and $51.44 \pm 2.39 \%$, respectively (Fig. 3A).

To further quantify the SSd-induced apoptotic effect, the treated cells were stained with Annexin V-FITC/PI and assayed using flow cytometry. A concentration-dependent increase in the percentages of necrotic (Annexin V-positive, PI-positive) and apoptotic (Annexin V-positive, PI-negative) cells was observed. Treatment of the DU145 cells with 3, 9 and $15 \mu \mathrm{M} \mathrm{SSd}$ for $12 \mathrm{~h}$ increased the rate of apoptosis from $7.37 \pm 2.39$ to $27.26 \pm 2.68,46.43 \pm 4.43$ and $75.77 \pm 3.01 \%$, respectively, with $<2 \%$ of cells being necrotic (Fig. 3B). Notably, $\sim 75 \%$ of the cells were apoptotic in the $15 \mu \mathrm{M} \mathrm{SSd}$ treatment group after $12 \mathrm{~h}$, with a concomitant $<10 \%$ increase in the G1 phase proportion (Fig. 2A). G1 phase arrest may only minimally account for the antiproliferative effect of SSd observed in DU145 cells. 
A

a

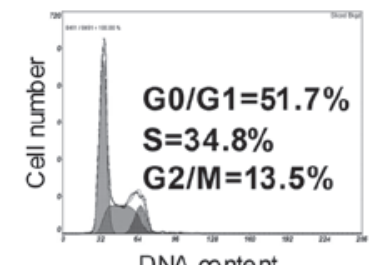

c

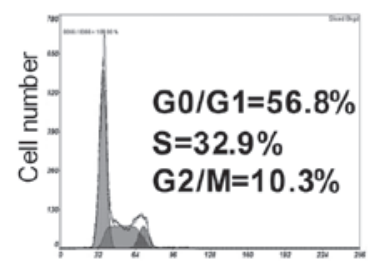

DNA œontent

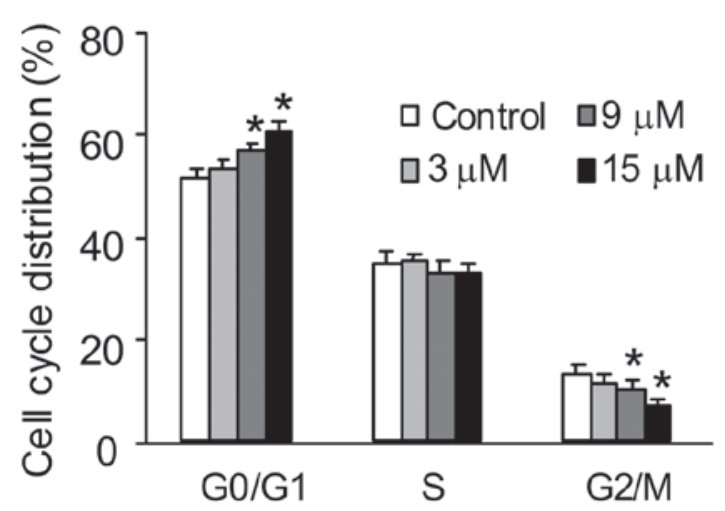

b

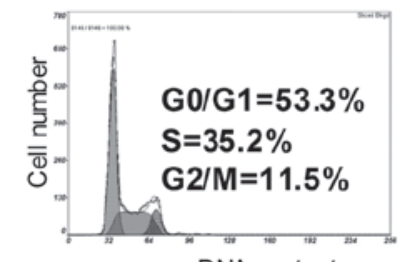

d

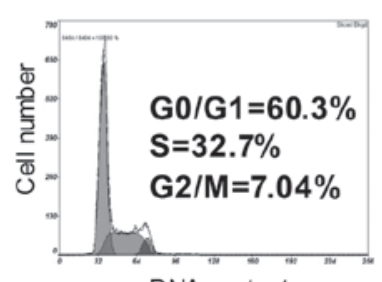

B
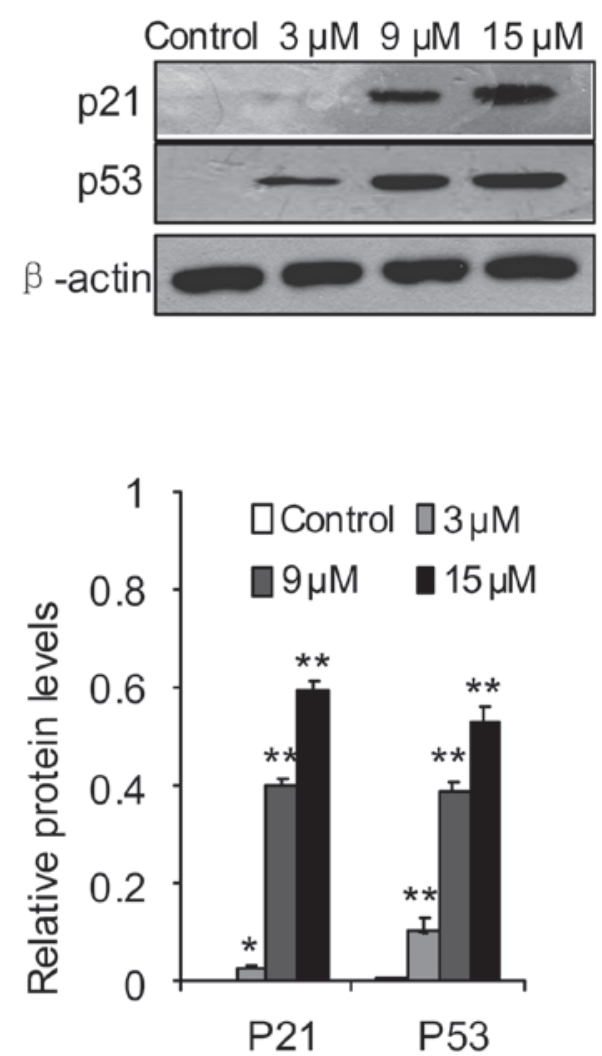

Figure 2. Effect of SSd on DU145 cell cycle distribution. (A) Flow cytometry results of the cell cycle phase distribution analysis. DU145 cells were treated with (a) control, or (b) $3 \mu \mathrm{M}$, (c) $9 \mu \mathrm{M}$ and (d) $15 \mu \mathrm{M} \mathrm{SSd}$ for $12 \mathrm{~h}$, and were stained with propidium iodide for flow cytometric analysis. The $\mathrm{x}$-axis and y-axis represent DNA content and number of cells, respectively. Data are expressed as the mean \pm standard error of three independent experiments with the similar results. ${ }^{*} \mathrm{P}<0.05 ;{ }^{* *} \mathrm{P}<0.01$ compared with the control. (B) Effect of SSd on the expression levels of p21 and p53. DU145 cells were treated with the indicated concentrations of SSd for $12 \mathrm{~h}$. The expression levels of p21 and p53 in DU145 cells were monitored using a western blot assay. $\beta$-actin was used as the loading control. The data shown are representative of three independent experiments with similar results. " $\mathrm{P}<0.05$; ${ }^{* *} \mathrm{P}<0.01$ compared with the control. SSd, saikosaponin-d.

Effect of SSd on ROS generation and MMP. The mitochondria-mediated intracellular signaling pathway is characterized by increased ROS generation, MMP dissipation and release of cytochrome $c$ from the mitochondria (25). A study indicated that SSd induced cellular ROS accumulation in cervical (HeLa and Siha), ovarian (SKOV3) and non-small cell lung (A549) cancer cell lines (9). Thus, the present study investigated the effect of SSd on the mitochondrial signaling pathway. ROS generation in DU145 cells was detected using a ROS Assay kit. As shown in Fig. 4A, following incubation with 3, 9 or $15 \mu \mathrm{M}$ SSd for $30 \mathrm{~min}$, the ROS levels in the SSd-treated group remained almost unchanged compared with those in the control group.

As depolarization of the MMP is a characteristic feature of apoptosis $(26,27)$, the MMP in DU145 cells was subsequently determined using Rho-123 staining and flow cytometry assay. The DU145 cells were exposed to different concentrations of SSd (3, 9 and $15 \mu \mathrm{M})$ for $12 \mathrm{~h}$ prior to Rho-123 staining. The results showed that SSd reduced the MMP in a concentration-dependent manner, from $98.15 \pm 1.84$ (in the DMSO control group) to $93.17 \pm 3.91,78.01 \pm 5.87$ and $22.21 \pm 3.41 \%$, respectively (Fig. 4B).
Effect of SSd on cytochrome c translocation and caspase-3 activation. Disruption of the MMP may lead to the release of cytochrome $c$ from the intermembrane space to the cytosol, which leads to the activation of procaspase-3 (28-30). To further define the apoptotic pathway, the levels of cytosolic cytochrome $c$ and activated caspase-3 in DU145 cells were determined. The western blotting results indicated that SSd increased the cytochrome $c$ levels in the cytosol and induced cleavage of caspase-3 (Fig. 5).

Effect of SSd on the expression levels of Bcl-2 and Bax. The Bcl-2 family proteins regulate apoptosis by controlling mitochondrial membrane stability (31). The Bcl-2 family contains anti-apoptotic, e.g. Bcl-2-associated agonist of cell death (Bad), BH3 interacting domain death agonist (Bid), Bax and Bcl-2-like 11 (apoptosis facilitator) (Bim), and pro-apoptotic, e.g. Bcl-2 and B-cell lymphoma-extra large (Bcl-xL), proteins, and the ratios of anti-apoptotic and pro-apoptotic proteins determine the fate of cells $(32,33)$. The present study investigated the effect of SSd on the expression levels of Bcl-2 and Bax. As shown in Fig. 6, SSd induced elevated levels of Bax, while it reduced levels of Bcl-2 in the DU145 cells compared with those in the control cells. 
A
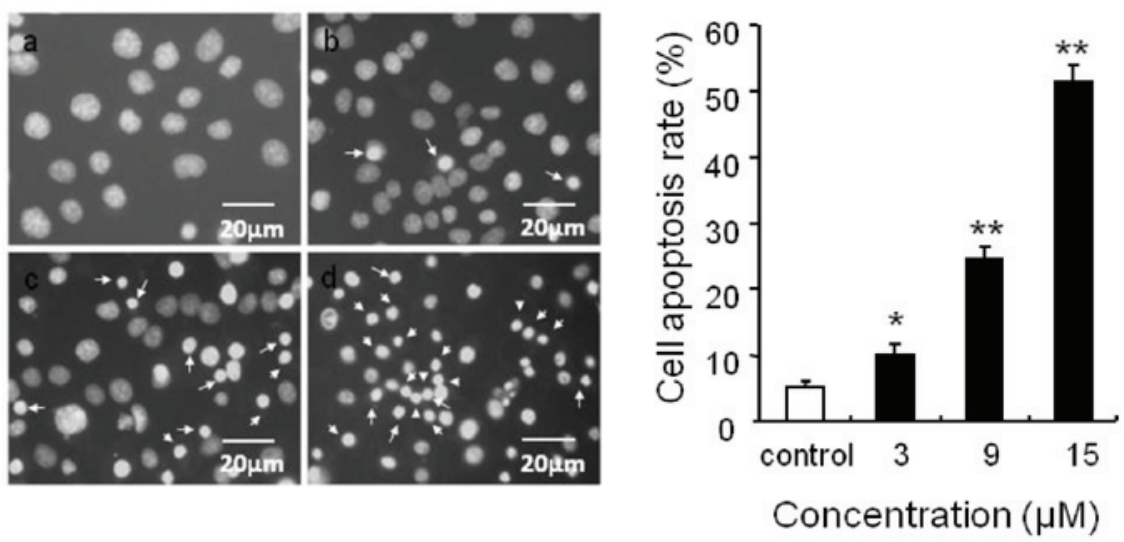

B

a

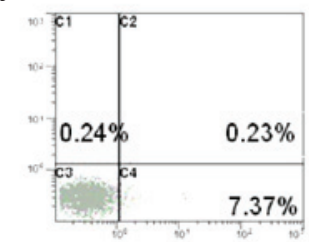

c

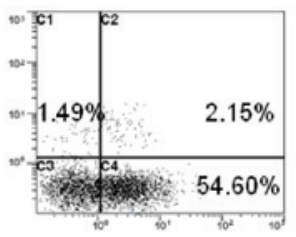

b

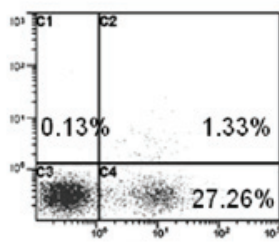

d

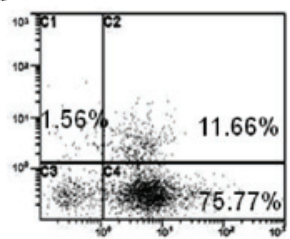

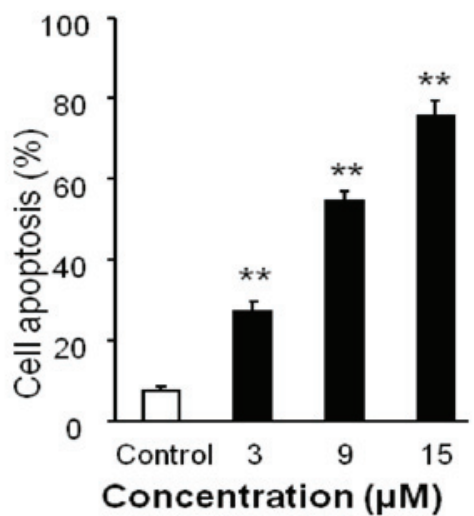

Figure 3. Induction of apoptosis of DU145 cells by SSd. (A) Cells were treated with (a) control, (b) $3 \mu \mathrm{M}$, (c) $9 \mu \mathrm{M}$ and (d) $15 \mu \mathrm{M}$ SSd for $2 \mathrm{~h}$, and stained with Hoechst 33258. The stained cells were observed under a fluorescence microscope. Arrows indicate the condensed and fragmented nuclei. Scale bar, $20 \mu \mathrm{m}$. Histogram shows the percentage of cleaved nuclei counted microscopically from 100 nuclei. Data are expressed as the mean \pm standard error of three independent experiments with the similar results. Magnification, x400. (B) DU145 cells treated with (a) control, or (b) $3 \mu \mathrm{M}$, (c) $9 \mu \mathrm{M}$ and (d) $15 \mu \mathrm{M}$ SSd for $12 \mathrm{~h}$. The cells were then stained with FITC-conjugated Annexin V and PI for flow cytometric analysis. The x-axis and y-axis represent Annexin V-FITC staining and PI, respectively. The cell populations shown in the lower right quadrant (Annexin $\left.\mathrm{V}^{+} / \mathrm{PI}\right)$ represent apoptotic cells, and those in the quadrant upper right $($ Annexin $\mathrm{V}^{+} / \mathrm{PI}^{+}$) represent necrotic cells. ${ }^{*} \mathrm{P}<0.05,{ }^{* *} \mathrm{P}<0.01$ compared with the control. SSd, saikosaponin-d; FITC, fluorescein isothiocyanate; PI, propidium iodide.

\section{Discussion}

Previous studies have reported that SSd inhibited proliferation of several cancer cell lines (8-11), while its effect on human prostate cancer cells remained under investigation. The present study revealed, for the first time to the best of our knowledge, that SSd induced apoptosis in the DU145 human prostate cancer cell line. The present study also provides insights into the mechanisms involved in SSd-induced apoptosis of DU145 cells.

The results of the present study demonstrated that SSd inhibited the proliferation of DU145 cells in a concentration-dependent manner. Nuclear fragmentation and chromosomal condensation are landmarks of apoptosis (34). The chromosomal condensation in the present study was confirmed by Hoechst 33258 staining. At the early stages of apoptosis, phosphatidyl serine (PS) is translocated from the inner face of the plasma membrane to the cell surface, while the cell membrane integrity decreases at late stages of apoptosis (35). Apoptotic and necrotic cells are discriminated using the PS-binding dye Annexin V-FITC and the DNA-binding dye PI. The Annexin V-FITC/PI double staining results of the present study revealed that $\mathrm{SSd}$ treatment induced apoptosis of DU145 cells in a concentration-dependent manner and $<2 \%$ of the treated cells were necrotic. Thus, the results suggested that the reduced cell viability in the MTT assay was due to apoptosis rather than necrosis.

Apoptosis is induced through two main pathways: The death receptor pathway and the intrinsic or mitochondrial pathway (29). A previous study showed that SS induced apoptosis via mitochondria-dependent and -independent pathways (11). As involvement of a mitochondria-mediated pathway in apoptosis is of notable value in the treatment of cancer (29), the effect of SSd on the mitochondrial apoptotic pathway was investigated in the present study. Depolarization of the MMP is a typical characteristic of apoptosis $(25,29)$; therefore, the MMP in DU145 cells was examined in the present study. The results revealed that the MMP was significantly dissipated following SSd treatment, which suggests the mitochondrial apoptotic pathway was induced by SSd.

The Bcl-2 family proteins, including anti-apoptotic (e.g. Bcl-2 and Bcl-xL) and proapoptotic (e.g. Bad, Bid, Bax and Bim) members, are key regulators in the mitochondrial apoptotic pathway (36). A slight change in the levels of these proteins may result in apoptosis (36). In the present study, SSd reduced the expression levels of Bcl-2 and increased the expression levels 
A
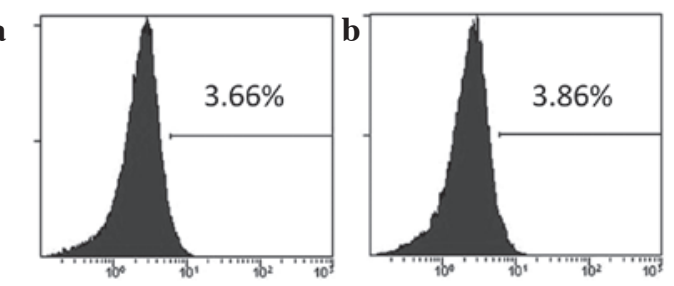

c
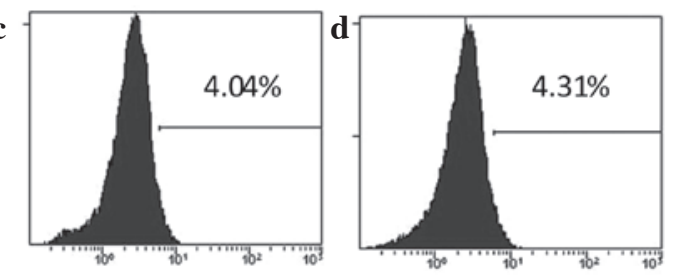

B
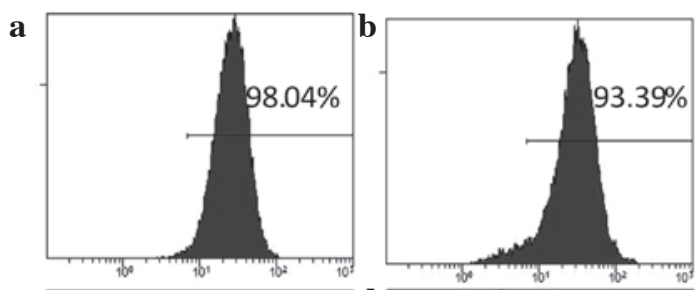

c

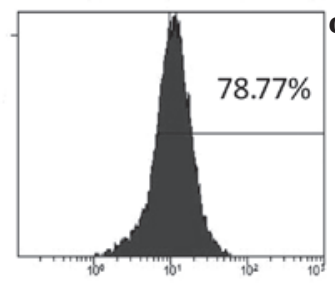

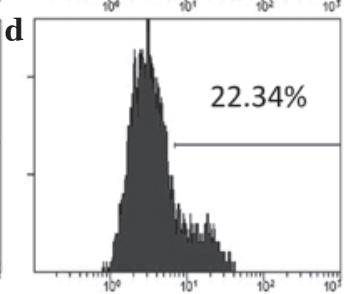
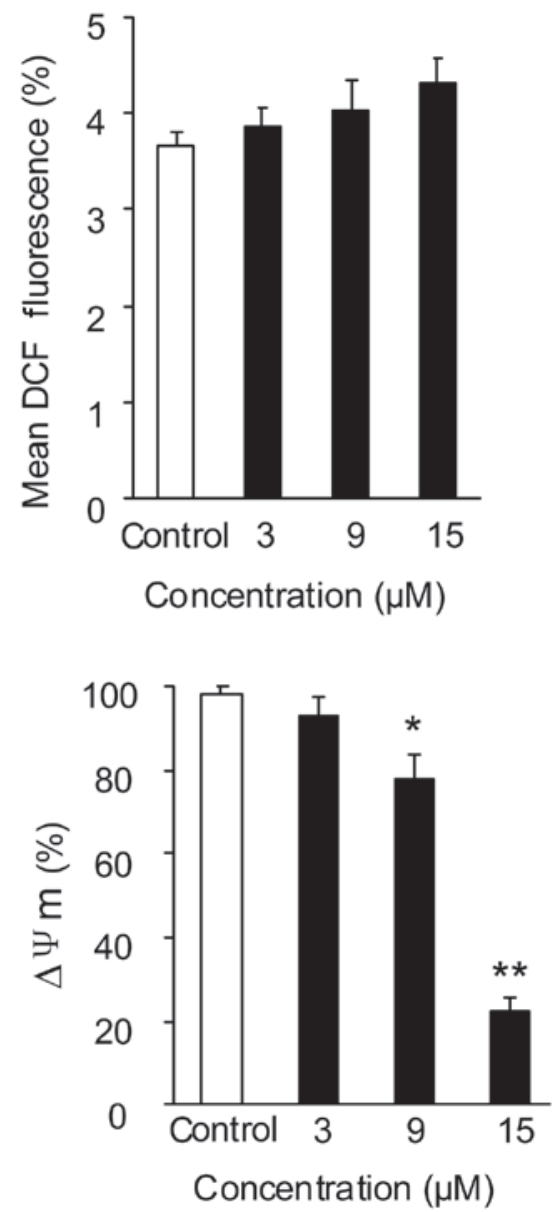

Figure 4. Flow cytometric analysis of ROS generation and the MMP in DU145 cells. (A) Effect ofSSD on ROS generation in DU145 cells. The DU145 cells were treated with (a) control, (b) $3 \mu \mathrm{M}$, (c) $9 \mu \mathrm{M}$ and (d) $15 \mu \mathrm{M}$ SSd for $30 \mathrm{~min}$. ROS generation was detected using a ROS assay kit. The data are expressed as the mean \pm standard error of three independent experiments with similar results. (B) Effect of SSd on the MMP levels in DU145 cells. DU145 cells were treated with (a) control, (b) $3 \mu \mathrm{M}$, (c) $9 \mu \mathrm{M}$ and (d) $15 \mu \mathrm{M} \mathrm{SSd}$ for $12 \mathrm{~h}$. The MMP levels were detected using the Rhodamine 123 staining method. Data are expressed as the mean \pm standard error of three independent experiments with similar results. ${ }^{*} \mathrm{P}<0.05,{ }^{* *} \mathrm{P}<0.01$ compared with the control. ROS, reactive oxygen species; MMP, mitochondrial membrane potential; SSd, saikosaponin-d.

A

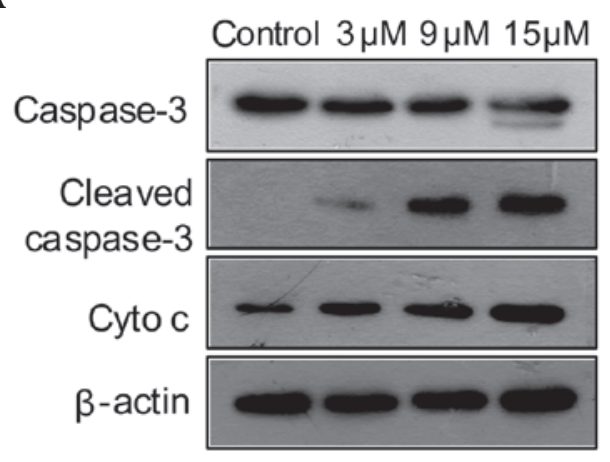

B

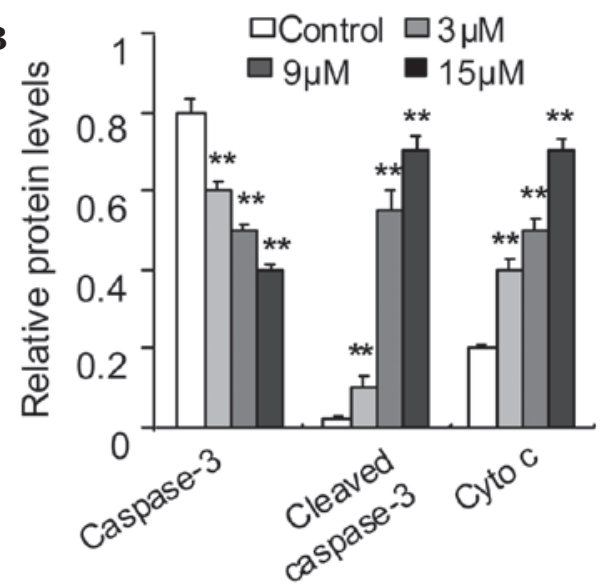

Figure 5. Effect of SSd on Cyto $c$ translocation and caspase-3 activation. (A) DU145 cells were treated with the indicated concentrations of SSd for 12 h. Cyto $c$ and cleaved caspase-3 in the DU145 cells were monitored using a western blot assay. $\beta$-actin was used as the loading control. (B) The data shown are representative of three independent experiments with the similar results. Data are represented as the mean \pm standard error. ${ }^{* *} \mathrm{P}<0.01$ compared with the control. SSd, saikosaponin-d; Cyto c, cytochrome c.

of Bax in a concentration-dependent manner, which resulted in an increased Bax/Bcl-2 ratio and thus may have triggered the mitochondrial pathway of apoptosis. Bcl-2 and Bax have roles in regulating cytochrome $c$ release $(31,37)$. Upon release 
A
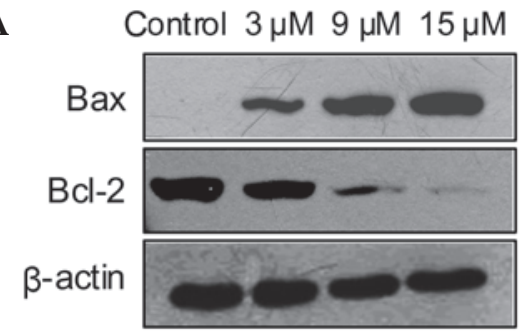

B

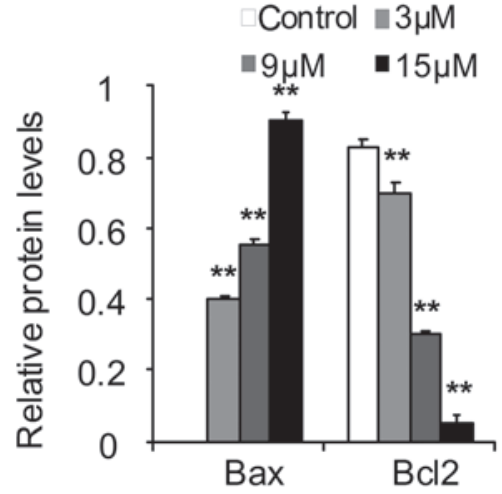

Figure 6. Effect of SS-d on Bcl-2 and Bax expression. (A) DU145 cells were treated with the indicated concentrations of SSd for $12 \mathrm{~h}$. Expression levels of Bax and Bcl-2 in the DU145 cells were monitored using a western blot assay. $\beta$-actin was used as the loading control. (B) The data shown are representative of three independent experiments with the similar results. Data are represented as the mean \pm standard error. ${ }^{* *} \mathrm{P}<0.01$ compared with the control. Bcl-2, B-cell lymphoma 2; Bax, Bcl-2-associated X protein; SSd, saikosaponin-d.

from the mitochondria, cytochrome $c$ activates the caspase cascade through the mitochondrial transition pore (32). The data of the present study revealed that SSd induced the release of cytochrome $c$ and activation of caspase-3. These results further support that SSd-induced apoptosis in DU145 cells via regulation of the $\mathrm{Bcl}-2$ family proteins.

Cytochrome $c$ release from mitochondria may result from overproduction of ROS $(38,39)$; thus, the effect of SSd on ROS production in DU145 cells was examined in the present study. The results showed that the ROS levels in SSd-treated DU145 cells remained unchanged compared with those in the control cells. SS compounds have long been used as antioxidants, and a previous study also confirmed the antioxidant activity of SSd in normal hepatocytes (40). Wang et al (9) reported that SSd induced cellular ROS accumulation in several types of cell line. The reason for the differences between the results of these studies remains to be determined, but may in part be due to the different cell lines used in the studies.

Inhibition of cell proliferation is implemented by cell cycle arrest. Although the data of the present study indicated that SSd treatment caused DU145 cell cycle arrest at G1 phase, the results suggest that apoptosis was the major reason for the inhibition of cell proliferation.

p21 (Cip1/Waf1), a major transcriptional target of p53 protein, has key roles in the transition from G1 phase into S phase $(41,42)$. p21 is a broad-specificity inhibitor of cyclin/cyclin-dependent kinase complexes; upregulation of p21 may lead to cell cycle arrest and inhibition of proliferation (43). Thus, the present study examined the expression levels of p21 and p53. The data showed that SSd significantly increased the levels of these two proteins compared with those in the control cells, which further supports the conclusion that SSd induces G1 arrest in DU145 cells.

In conclusion, the present study revealed that SSd-induced mitochondrial dysfunction is the major reason for the induction of apoptotic cell death in DU145 human prostate cancer cells treated with SSd. The induction of apoptosis was associated with dissipation of the MMP, release of cytochrome $c$, activation of caspase- 3 and modulation of $\mathrm{Bcl}-2$ family proteins. G0/G1 phase arrest is a minor reason for the SSd-induced inhibition of cell proliferation; this effect was associated with upregulation of the levels of p53 and p21. Further in-depth studies are required to examine SSd-induced apoptosis and cell cycle perturbation in DU145 cells. SSd may be developed into a leading candidate drug for prostate cancer therapy.

\section{Acknowledgements}

This study was supported by the National Clinical Key Specialty Construction Project (no. 2011-873).

\section{References}

1. Lee J, Yang DH, Suh JH, Kim U, Eom HY, Kim J, Lee MY, Kim J and Han SB: Species discrimination of Radix Bupleuri through the simultaneous determination of ten saikosaponins by high performance liquid chromatography with evaporative light scattering detection and electrospray ionization mass spectrometry. J Chromatogr B Analyt Technol Biomed Life Sci 879: 3887-3895, 2011.

2. Kato M,Pu MY, Isobe K, Iwamoto T, Nagase F, Lwin T, Zhang YH, Hattori T, Yanagita N and Nakashima I: Characterization of the immunoregulatory action of saikosaponin-d. Cell Immunol 159: $15-25,1994$.

3. Kato $\mathrm{M}, \mathrm{Pu} \mathrm{MY}$, Isobe $\mathrm{K}$, Hattori $\mathrm{T}$, Yanagita $\mathrm{N}$ and Nakashima I: Cell type-oriented differential modulatory actions of saikosaponin-d on growth responses and DNA fragmentation of lymphocytes triggered by receptor-mediated and receptor-bypassed pathways. Immunopharmacology 29: 207-213, 1995.

4. Chang JS, Wang KC, Liu HW, Chen MC, Chiang LC and Lin CC: Sho-saiko-to (Xiao-Chai-Hu-Tang) and crude saikosaponins inhibit hepatitis B virus in a stable HBV-producing cell line. Am J Chin Med 35: 341-351, 2007.

5. Ohtake N, Nakai Y, Yamamoto M, Sakakibara I, Takeda S, Amagaya $\mathrm{S}$ and Aburada M: Separation and isolation methods for analysis of the active principles of Sho-saiko-to (SST) oriental medicine. J Chromatogr B Analyt Technol Biomed Life Sci 812: 135-148, 2004.

6. Bao Y, Li C, Shen H and Nan F: Determination of saikosaponin derivatives in Radix bupleuri and in pharmaceuticals of the chinese multiherb remedy xiaochaihu-tang using liquid chromatographic tandem mass spectrometry. Anal Chem 76: 4208-4216, 2004.

7. Wu GC, Wu H, Fan LY and Pan HF: Saikosaponins: a potential treatment option for systemic lupus erythematosus. Ir J Med Sci 180: 259-261,2011.

8. Hsu YL, Kuo PL, Chiang LC and Lin CC: Involvement of p53, nuclear factor kappaB and Fas/Fas ligand in induction of apoptosis and cell cycle arrest by saikosaponin $\mathrm{d}$ in human hepatoma cell lines. Cancer Lett 213: 213-221, 2004.

9. Wang Q, Zheng XL, Yang L, Shi F, Gao LB, Zhong YJ, Sun H, He F, Lin Y and Wang X: Reactive oxygen species-mediated apoptosis contributes to chemosensitization effect of saikosaponins on cisplatin-induced cytotoxicity in cancer cells. J Exp Clin Cancer Res 29: 159, 2010.

10. Hsu YL, Kuo PL and Lin CC: The proliferative inhibition and apoptotic mechanism of Saikosaponin D in human non-small cell lung cancer A549 cells. Life Sci 75: 1231-1242, 2004. 
11. Kim BM and Hong SH: Sequential caspase-2 and caspase- 8 activation is essential for saikosaponin a-induced apoptosis of human colon carcinoma cell lines. Apoptosis 16: 184-197, 2011.

12. Chen JC, Chang NW, Chung JG and Chen KC: Saikosaponin-A induces apoptotic mechanism in human breast MDA-MB-231 and MCF-7 cancer cells. Am J Chin Med 31: 363-377, 2003.

13. Zong Z, Fujikawa-Yamamoto K, Tanino M, Teraoka K, Yamagishi $\mathrm{H}$ and Odashima S: Saikosaponin b2-induced apoptosis of cultured B16 melanoma cell line through down-regulation of PKC activity. Biochem Biophys Res Commun 219: 480-485, 1996

14. American Cancer Society. [http://www.cancer.org/cancer/prostatecancer/overviewguide/prostate-cancer-overview-key-statistics].

15. Marta GN, Hanna SA, Fernandes da Silva JL and Carvalho Hde A Screening for prostate cancer: an updated review. Expert Rev Anticancer Ther 13: 101-108, 2013.

16. Kollmeier MA and Zelefsky MJ: How to select the optimal therapy for early-stage prostate cancer. Crit Rev Oncol Hematol 84 (Suppl 1): e6-e15, 2012.

17. Drudge-Coates L and Turner B: Prostate cancer overview. Part 1: non-metastatic disease. Br J Nurs 21: S23-S28, 2012.

18. Drudge-Coates L and Turner B: Prostate cancer overview. Part 2 metastatic prostate cancer. Br J Nurs 21: S23-S24, S26-S28, 2012.

19. Twentyman PR and Luscombe M: A study of some variables in a tetrazolium dye (MTT) based assay for cell growth and chemosensitivity. Br J Cancer 56: 279-285, 1987.

20. Fuchs Y and Steller H: Programmed cell death in animal development and disease. Cell 147: 742-758, 2011.

21. Rhind N and Russell P: Signaling pathways that regulate cell division. Cold Spring Harb Perspect Biol 4: a005942, 2012.

22. Dash BC and El-Deiry WS: Cell cycle checkpoint control mechanisms that can be disrupted in cancer. Methods Mol Biol 280 99-161, 2004.

23. Kitazumi I and Tsukahara M: Regulation of DNA fragmentation: the role of caspases and phosphorylation. FEBS J 278: 427-441, 2011.

24. Zimmermann KC, Bonzon C and Green DR: The machinery of programmed cell death. Pharmacol Ther 92: 57-70, 2001.

25. Mignotte B and Vayssiere JL: Mitochondria and apoptosis. Eur J Biochem 252: 1-15, 1998.

26. Armstrong JS: Mitochondria: a target for cancer therapy. $\mathrm{Br}$ J Pharmacol 147: 239-248, 2006.
27. Bras M, Queenan B and Susin SA: Programmed cell death via mitochondria: different modes of dying. Biochemistry (Mosc) 70: 231-239, 2005.

28. Brenner D and Mak TW: Mitochondrial cell death effectors. Curr Opin Cell Biol 21: 871-877, 2009.

29. Chalah A and Khosravi-Far R: The mitochondrial death pathway. Adv Exp Med Biol 615: 25-45, 2008.

30. Pradelli LA, Bénéteau M and Ricci JE: Mitochondrial control of caspase-dependent and -independent cell death. Cell Mol Life Sci 67: 1589-1597, 2010.

31. Ola MS, Nawaz M and Ahsan $\mathrm{H}$ : Role of Bcl-2 family proteins and caspases in the regulation of apoptosis. Mol Cell Biochem 351: 41-58, 2011.

32. García-Sáez AJ: The secrets of the Bcl-2 family. Cell Death Differ 19: 1733-1740, 2012.

33. Estaquier J, Vallette F, Vayssiere JL and Mignotte B: The mitochondrial pathways of apoptosis. Adv Exp Med Biol 942: 157-183, 2012.

34. Robertson JD, Orrenius S and Zhivotovsky B: Review: nuclear events in apoptosis. J Struct Biol 129: 346-358, 2000.

35. Wlodkowic D, Skommer J and Darzynkiewicz Z: Cytometry of apoptosis. Historical perspective and new advances. Exp Oncol 34: 255-262, 2012.

36. Zinkel S, Gross A and Yang E: BCL2 family in DNA damage and cell cycle control. Cell Death Differ 13: 1351-1359, 2006.

37. Burlacu A: Regulation of apoptosis by Bcl-2 family proteins. J Cell Mol Med 7: 249-257, 2003.

38. Schultz DR and Harrington WJ Jr: Apoptosis: programmed cell death at a molecular level. Semin Arthritis Rheum 32: 345-369, 2003.

39. Gottlieb RA: Mitochondria and apoptosis. Biol Signals Recept 10: 147-161, 2001.

40. Fan J, Li X, Li P, Li N, Wang T, Shen H, Siow Y, Choy P and Gong Y: Saikosaponin-d attenuates the development of liver fibrosis by preventing hepatocyte injury. Biochem Cell Biol 85: 189-195, 2007.

41. Besson A, Dowdy SF and Roberts JM: CDK inhibitors: cell cycle regulators and beyond. Dev Cell 14: 159-169, 2008.

42. Junttila MR and Evan GI: p53 - a Jack of all trades but master of none. Nat Rev Cancer 9: 821-829, 2009.

43. Abbas T and Dutta A: p21 in cancer: intricate networks and multiple activities. Nat Rev Cancer 9: 400-414, 2009. 